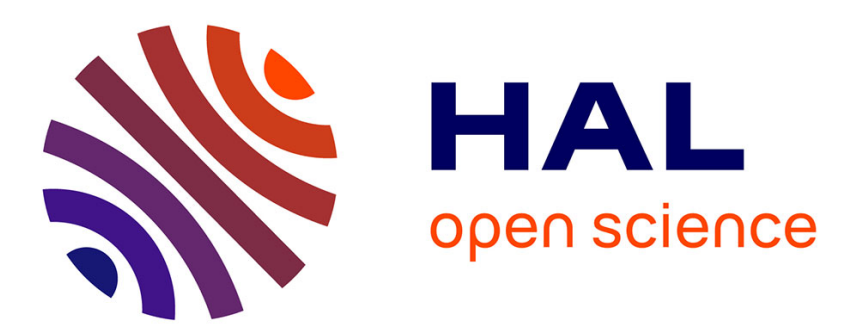

\title{
Maximizing player's anticipation by applying the proximity-compatibility principle to the design of video games
}

Loïc Caroux, Ludovic Le Bigot, Nicolas Vibert

\section{To cite this version:}

Loïc Caroux, Ludovic Le Bigot, Nicolas Vibert. Maximizing player's anticipation by applying the proximity-compatibility principle to the design of video games. Human Factors, 2011, 53, pp.103-117. 10.1177/0018720811400600 . hal-01470733

\section{HAL Id: hal-01470733 \\ https://hal.science/hal-01470733}

Submitted on 17 Feb 2017

HAL is a multi-disciplinary open access archive for the deposit and dissemination of scientific research documents, whether they are published or not. The documents may come from teaching and research institutions in France or abroad, or from public or private research centers.
L'archive ouverte pluridisciplinaire HAL, est destinée au dépôt et à la diffusion de documents scientifiques de niveau recherche, publiés ou non, émanant des établissements d'enseignement et de recherche français ou étrangers, des laboratoires publics ou privés. 
Rapport technique : 2011/01/L.CAR

\section{Maximizing player's anticipation by applying the proximity-compatibility principle to the design of video games}

Loïc CAROUX, Ludovic LE BIGOT, Nicolas VIBERT - Centre de Recherches sur la Cognition et l'Apprentissage (CeRCA), CNRS UMR 6234 - University of Poitiers University François Rabelais of Tours, Maison des Sciences de l'Homme et de la Société, Poitiers, France.

To appear in :

Caroux, L., Le Bigot, L., \& Vibert, N.. Maximizing player's anticipation by applying the proximity-compatibility principle to the design of video games. Human Factors.

Address for correspondence to the first author at:

Loïc CAROUX,

Université de Poitiers -CNRS

Centre de Recherches sur la Cognition et l'Apprentissage (CeRCA) - CNRS UMR 6234

MSHS - Bâtiment A5 - 5 rue Théodore Lefebvre - 86000 Poitiers, France

86000 Poitiers

France

E-mail : loic.caroux@univ-poitiers.fr

Tel : 33 (0)5.49.45.46.82 ou 46.10

Fax : $33(0) 5.49 .45 .46 .16$ 
KEYWORDS: interactive environments, dynamic visual displays, multiple resources model, moving visual background.

\section{ABSTRACT}

Objective. Two experiments were conducted to investigate elements of the spatial design of video game interfaces.

Background. In most video games, both the objects and the background scene are moving. Players must pay attention to what appears in the background to anticipate events while looking at head-up displays. According to the proximitycompatibility principle, game-related information should be put as close as possible to the anticipation zone.

Method. Participants played a video game where they had to anticipate the upward movement of obstacles. The score location was manipulated. The average vertical gaze position and dispersion were used to assess anticipation and extent of visual scanning, respectively.

Results. Putting the score at the bottom rather than the top of the game window, within the anticipation zone, was expected to minimize attentional moves. Experiment 1 revealed lower average gaze positions and reduced extent of visual scanning in that condition, but the score performance did not improve significantly. Experiment 2 demonstrated that players' performance increased compared to the bottom condition when the score was displayed just below but outside the game window, despite an increased extent of visual scanning.

Conclusion. Positioning the score just outside the anticipation zone facilitated anticipation of the movement of obstacles and led to better performance than when the score overlapped with the game anticipation zone.

Application. For games requiring visual anticipation, contextual information should be located in the direction of anticipation, but not within the anticipation zone. This recommendation complements the proximity compatibility principle for simple dynamic displays.

\section{INTRODUCTION}

\section{HUMAN FACTORS IN VIDEO GAMES}

Since computerized video games first appeared, their design has strongly evolved. Video games include more and more realistic visual scenes that involve complex human-computer interactions. Because of the high complexity of current video games, using a player-centered design has become essential for commercial success (Pagulayan, Keeker, Wixon, Romero, \& Fuller, 2008). From the perspective of human factors, video games are different from other computer applications like web pages or productivity software (Pagulayan et al.) in that they are virtual environments in which the visual stimuli get the player to interact with an alternative world, which may or may not mimic the real world (Stanney, Mollaghasemi, Reeves, Breaux, \& Graeber, 2003). In most of these environments, both the objects in the visual scene (e.g., avatar, enemies or targets) and the visual scene background (e.g., patterns, building insides or landscapes) are moving. In addition, a head-up display that gives information linked to the current game situation is superimposed on the action scene. Generally, permanent information such as the current score, health points or maps of the virtual world is displayed on peripheral areas of the game screen. Non-permanent information such as warning messages may be displayed on the main game area. Contrary to the head-up displays used in other environments, video games' head-up displays are usually opaque and may thus mask elements of the game display. These elements generate complex, dynamic visual displays for which visual perception rules might differ from those of static scenes. 
Guidelines and heuristics are the most used tools to design human-computer interfaces for Web pages and productivity software considering a user-centered point of view (e.g., Nielsen, 1993). In most cases, the video game industry works with heuristics that were adapted from these ergonomic guidelines. However, the particularities of the virtual dynamic environments used in video games call for a greater specification of heuristics (Barr, Noble, \& Biddle, 2007). In particular, game designers could adapt the display design guidelines that emerged from human factors research on dynamic human-computer systems (e.g., modern plane cockpits or flight simulators) such as the proximity-compatibility principle (PCP) proposed by Wickens and Carswell (1995). The aim of the current article was to elaborate on the PCP to propose and validate a new heuristic for the spatial design of video games interfaces using an experimental method based on cognitive ergonomics theories.

\section{SPLIT-ATTENTION}

Multi-task environments. Interacting with complex systems like video games involves many different cognitive processes (Boot, Kramer, Simons, Fabiani, \& Gratton, 2008), among which attention plays a prominent role (Green \& Bavelier, 2003). The player must pay attention to several auditory and visual information sources while controlling manually or vocally various elements of the game. The multiplicity of information inputs and outputs creates a multitask environment in which the player's attentional capacity can be easily overloaded.

Each task that the player must perform requires attentional resources. The addition of multiple demands may generate conflicts in the allocation of these resources and thus decrease a player's performance. Models of attention generally assume a single pool of attentional resources, which can be shared in a flexible way between modalities and processes. As such, they can be used to explain interference between tasks within such environments. The multiple-resources model proposed by Wickens $(1984,2002)$, for example, gives a frame to explain and predict how attentional resources are allocated when multiple tasks must be performed simultaneously. Wickens argued that a "limited capacity central processor" was shared according to both the qualitative features and respective difficulty of the tasks.

The multiple resources model. The multiple resources model considers four dichotomous dimensions to categorize the attentional demands of a task (for review, Wickens \& McCarley, 2008): the processing stage (information encoding and processing stage versus motor response stage), the perceptual modality (visual versus auditory), the processing code (spatial or analog versus verbal or symbolic) and finally the processing mode within the visual modality (focal versus ambient processing). There is indeed good evidence that focal versus ambient visual processing involve separate resources (Wickens, 2002), and this last dimension is particularly important within the context of visual displays. The resources of the focal channel, which generally involves central vision, would be allocated to the finest attentional tasks such as object recognition. The resources of the ambient channel, which involves peripheral vision, would be used for more general tasks like ego-motion, orientation perception, or to detect changes in the peripheral visual field. Numerous visual search and reading studies have demonstrated that information processed by peripheral vision was used to guide eye movements within visual displays (Rayner, 1998).

The attentional resources needed for a task can be characterized along each of the four dimensions described above. In dual-task situations, the more the tasks use resources from common levels in one or more dimensions, the more this resource sharing provokes interference. In contrast, there should be less interference between attentional demands that tap resources from distant levels in all dimensions. Hence, the model can predict the relative amounts of interference between simultaneously performed tasks within high-workload environments.

\section{INTEGRATING INFORMATION}

Video gaming requires the simultaneous performance of various tasks (Ang, Zaphiris, \& Mahmood, 2007), during which the player must divide his or her attention between different auditory or visual information sources, acquire the necessary information and integrate that information. Current studies investigating the optimal design for complex multimodal displays (Wickens \& McCarley, 2008) suggest that they should minimize the cost of information integration. 
The displays should facilitate the mental combination of information from separate sources, particularly when attention is divided.

The proximity-compatibility principle. The PCP can be used as guidelines to facilitate information integration. Wickens and Carswell (1995) demonstrated that two information sources requiring divided attention in service of integration during a common task or mental operation should be put close to each other in the display. In contrast, pieces of information used in isolation can be put further apart. One of the ways to increase proximity between information sources is to maximize their spatial proximity and put them next to each other. This reduces the amplitude of attentional moves in divided attention situations. For example, Renshaw, Finlay, Tyfa, and Ward (2004) studied an information search task within a graph that required the integration of multiple information sources. They showed that the participants responded faster when the information sources (e.g. curve label, numeral data) were displayed within the graph rather than outside as legends.

However, close spatial proximity between visual signals does not always lead to better information integration. First, these conditions can lead to confusion or masking, e.g., when a head-up display is superimposed on a main scene view. This can imply a negative effect or a reduced benefit to user performance (Wickens et al., 2004). Second, the PCP continuum (Wickens \& Carswell, 1995; Wickens \& McCarley, 2008) emphasizes that the less information sources are used simultaneously for the same task, the more spatial proximity between them can lead to interference; as a result, they should be placed with less proximity. In the most extreme cases, the continuum extends from tasks where complete integration of the different pieces of information is required to tasks where the information sources are never used simultaneously. In between, there are cases where only some features of the two pieces of information must be combined, or where both information must be used simultaneously without being combined or integrated.

Visual anticipation of information processing in video games. In video games, anticipating information appearance or modification is essential. Both the visual objects involved in the action and the scene background are generally moving, and the information given by head-up displays is often modified "in real time". As such, to perform well, the player must both anticipate the movements and detect changes as fast as possible.

When the scene background is moving in one direction, for example upwards, to give the illusion of self-movement, the player must continuously pay attention to the new visual input that appears at the bottom of the screen to detect potential obstacles, friends or enemies as quickly as possible. At the same time, he or she must go on playing the game and looking at or listening to the information given by the system. Although visual anticipation by video gamers has not received empirical attention, similar situations are encountered by human operators within other virtual or real environments. Driving and flying are among the best examples of such tasks. Drivers and pilots must pay attention to multiple sources of information while anticipating events by looking ahead to their destination. Eye-tracking methods were used to observe gaze movements and evaluate the operators' degree of anticipation (e.g., Underwood, 2007; Underwood, Chapman, Brocklehurst, Underwood, \& Crundall, 2003; Wickens et al., 2003). For example, Underwood et al. (2003) studied the eye movements of novice and expert drivers. In natural driving situations, expert drivers paid more attention to more distal road locations than to other areas of the environment, which was taken as evidence that they spent most of their time anticipating possible hazards. Novice drivers less frequently displayed these patterns, suggesting that more attentional resources can be devoted to anticipation when driving becomes more automatic.

The framework of the "Salience, Effort, Expectancy and Value" (SEEV) model (Wickens et al., 2003; Wickens \& McCarley, 2008) can help predict users' eye movements on the Areas of Interest (AOI) of dynamic visual displays. The model claims that the probability of attending to each particular AOI results from four factors. The visual salience of the area and the effort needed to access the information (i.e. the distance between the $\mathrm{AOI}$ and other information sources) depend on the physical characteristics of the display. The two additional factors are the user's expectancy to find relevant information in each $\mathrm{AOI}$ (which is linked to the frequency of information changes in this area) and the value of the information for the user. For example, Horrey, Wickens, and Consalus (2006) predicted drivers' eye movements between the outside world and in-vehicle technology as a function of task priority and the rate of information change within the two areas. 


\section{THE CURRENT STUDY}

The first goal of the present study was to characterize the eye movements of video game players who must anticipate their actions by looking towards the place where the moving background scene originates. They must also combine this continually renewed visual information with other information available on the screen, e.g., the score they have reached. Accordingly, a second goal of the study was to evaluate the impact of spatial proximity between the anticipation zone (defined as the part of the main game area where new moving items such as obstacles appear) and the location of screen-displayed information on both the anticipation behavior and game performance. Indeed, players' attention to the anticipation zone should result in better game performance.

In the two experiments, the location on the screen of a video game head-up display, namely the score, was manipulated. The video game that was used had simple rules and a minimalist, two-dimensional graphic design. It was composed of a horizontally moving ball controlled by the player, and of square-like obstacles that continuously appeared at the bottom of the screen and moved upwards to disappear at the top. The head-up display was composed of one element only, the score. The player had to visually anticipate the movements of obstacles in order to avoid touching them with the ball while following the evolution of the score display. The score automatically increased with time as long as the ball did not touch any obstacle, but the game was lost as soon as the ball touched one.

A first, preliminary hypothesis was that all elements of the display (the ball, the moving obstacles, and the score display) are relevant for the game task and are thus scanned by participants while playing. In accordance with previous research (e.g., Underwood et al., 2003), which showed that drivers made most of their fixations on road anticipation areas, the second hypothesis was that video game players make more fixations on the areas below than on the areas above the ball axis of movement to anticipate the movement of obstacles. The third hypothesis was that a score display location at the bottom (rather than the top of the screen) reduces the distance between the score display and the game area that must be scanned to anticipate obstacles (game anticipation zone), and thus increases the anticipation of their movement. In alignment with the PCP (Wickens \& Carswell, 1995; Wickens \& McCarley, 2008), the fourth hypothesis was that players would perform better when the score is displayed at the bottom rather than at the top of the screen due to a reduced extent of visual scanning and increased anticipation. Table 1 displays the main characteristics of the score display designs used in the "top" and "bottom" conditions of Experiment 1.

Table 1. Summary of the main characteristics of the four score display conditions: "Top", "bottom" (Experiments 1 and 2), "just above the ball" and "far bottom" (Experiment 2).

\begin{tabular}{lllll} 
Condition & Top & Bottom & Just above the ball & Far bottom \\
\hline $\begin{array}{l}\text { Location of the } \\
\text { score box }\end{array}$ & $\begin{array}{l}\text { At the top of the } \\
\text { game screen }\end{array}$ & $\begin{array}{l}\text { At the bottom of the } \\
\text { game screen }\end{array}$ & $\begin{array}{l}\text { In the middle of the } \\
\text { game screen, just } \\
\text { above the ball's axis } \\
\text { of movement }\end{array}$ & $\begin{array}{l}\text { Just below the } \\
\text { game screen }\end{array}$ \\
$\begin{array}{l}\text { Distance between } \\
\text { the score box and } \\
\text { main game area }\end{array}$ & $\begin{array}{l}\text { Far above } \\
\text { (low proximity) }\end{array}$ & $\begin{array}{l}\text { Within the main } \\
\text { game area } \\
\text { (high proximity) }\end{array}$ & $\begin{array}{l}\text { Just above } \\
\text { (intermediate } \\
\text { proximity) }\end{array}$ & $\begin{array}{l}\text { Just below } \\
\text { (intermediate } \\
\text { Cluttering at the } \\
\text { score box locationity) }\end{array}$ \\
& $\begin{array}{l}\text { Intermediate : Some } \\
\text { obstacles are masked, } \\
\text { but outside the } \\
\text { anticipation zone }\end{array}$ & $\begin{array}{l}\text { High : The score box } \\
\text { is masking some } \\
\text { obstacles within the } \\
\text { anticipation zone }\end{array}$ & $\begin{array}{l}\text { Intermediate : Some } \\
\text { obstacles are masked, } \\
\text { but outside the } \\
\text { anticipation zone }\end{array}$ & $\begin{array}{l}\text { Low }: \text { No } \\
\text { obstacles are } \\
\text { masked. }\end{array}$ \\
\hline
\end{tabular}




\section{EXPERIMENT 1}

\section{METHODS}

Participants. Thirty right-handed volunteers (10 women, 20 men) took part in the experiment. Their average age was 22.9 years $(S D=3.2)$ and their average length of schooling 15.80 years $(S D=2.35)$. Eight of the participants had never played video games, ten participants played or were playing occasionally (less than 2 hours a week) and twelve were regular players (more than 2 hours a week).

Material and apparatus. A game was created with the "Game Maker 7.0" software. The rule was to "avoid" obstacles moving towards a ball. The obstacles were black and dark gray squares of $32 \times 32$ pixels size $(11 \times 11 \mathrm{~mm})$ that scrolled up the game window at a 150 pixels $(51 \mathrm{~mm})$ per second speed on a light gray background. The game window did not cover the whole screen, but was 480 pixels wide by 672 pixels high and covered a $162 \times 227$ millimeters area on the screen of the eye-tracker. About fifty obstacles were visible at the same time. Two or three obstacles appeared every 210 milliseconds at the bottom of the game window, at random locations on the same horizontal line. A green ball located in the middle of the window could be moved by the player through a keypad, on the horizontal axis only. The ball was controlled in position and moved at a constant speed of 210 pixels (about 6.2 degrees of visual angle) per second in the left or right direction as long as the "left" or "right" key of the keypad was pressed. The vertical distance between the bottom tip of the ball and the bottom of the game screen was 320 pixels (108 mm, 9.4 degrees of visual angle). Hence, the ball could be moved along the entire width of the game screen in $2210 \mathrm{~ms}$, while the obstacles took 2130 ms to move up from the bottom of the game screen to the ball axis of movement. The game was lost as soon as the ball touched an obstacle.

A score was displayed in a blue rectangle in either one of two locations, namely the top-right and bottom-right corners of the game window ("top" and "bottom" conditions, Figure 1). At the beginning of each trial, the score was set to 0 and increased by 1 point for each line of obstacles that was avoided, which means that it increased at a rate of 1 point each $210 \mathrm{~ms}$ as long as the ball did not touch any square.

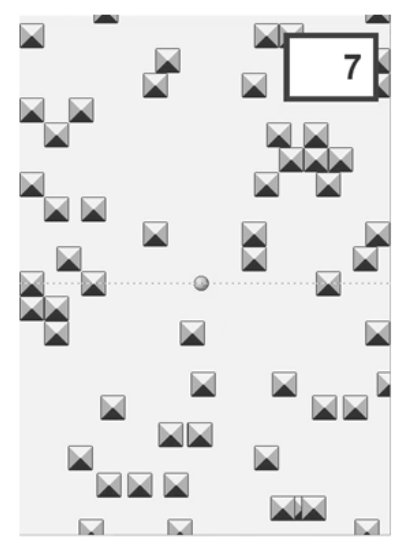

Top

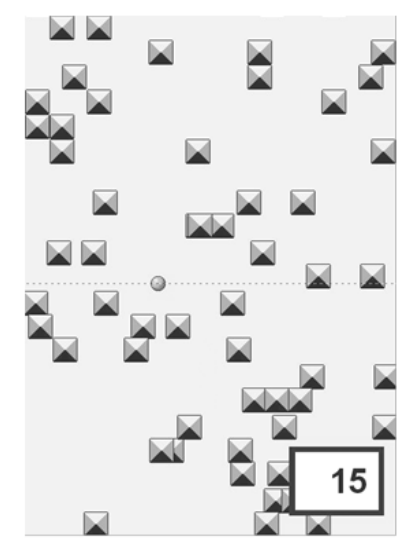

Bottom

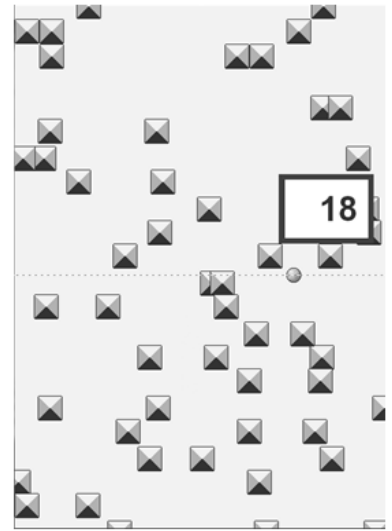

Just above the ball

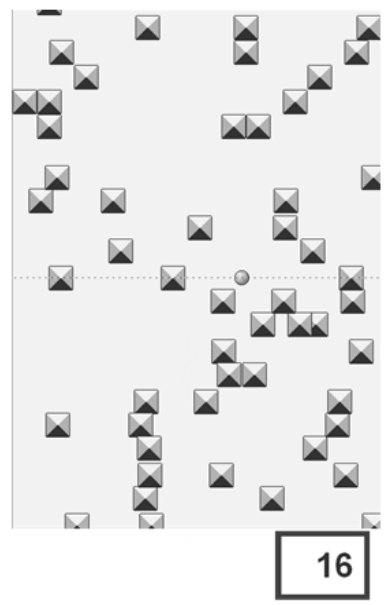

Far bottom

Figure 1. Screenshots of the game displays in the four conditions of score locations. Only the "top" and "bottom" locations were manipulated between-participants in Experiment 1. The four conditions were manipulated within-participants in Experiment 2. 
A non-intrusive, head free Tobii 1750 eye-tracker that resembled a 17" computer screen (1024x768 pixels resolution) was used to assess natural game conditions. The eye tracker was controlled via a Fujitsu-Siemens laptop which collected the data and ran the video game program. Gaze positions were obtained at a $50 \mathrm{~Hz}$ frequency with an average precision of 0.5 degree of visual angle. Since the average distance between the eye-tracker screen and participants' eyes was $660 \mathrm{~mm}(S D=66), 0.5$ degree of visual angle corresponded to about $5.75 \mathrm{~mm}$ and 17 pixels on the screen. Eye movements were analyzed using the ClearView 2.7.0 software. Eye fixations were defined as any period where gaze stayed for $60 \mathrm{~ms}$ (three successive gaze points at $50 \mathrm{~Hz}$ ) or more within a 30 pixels ( $0.89 \mathrm{deg}$ of visual angle) diameter area.

Design and procedure. The location of the score display was manipulated between-participants. The participants were randomly assigned to one of the two conditions provided there was no significant difference in the distribution of video gaming expertise between groups. Five non players, three occasional players and seven regular players played in the "bottom" condition, while three non players, seven occasional players and five regular players played in the "top" condition.

The initial goal of each participant was to hit a threshold score of 100 points on one trial, but participants were not given any instruction to follow the score display. They could try as many times as wanted to reach the goal, but the total time of effective play (namely the summed duration of all trials) was limited to ten minutes, even if the 100 points threshold score was never hit.

The participant began each trial by pushing an "enter" key. The ball, moving obstacles and score appeared, and the participant could control the ball through the "left" and "right" keys to avoid the upward moving obstacles. The score increased with time as long as obstacles were avoided, but the trial stopped as soon as the ball touched one of the squares, and all the elements of the game display disappeared. The score was recorded and then reinitialized to 0 for the next trial. When a participant hit the 100 points threshold score on one trial, the game went on as usual until the ball touched an obstacle. A new threshold score 50 points higher (i.e. 150 points) was assigned to the participant for the next trials. If the participant hit this new threshold score before having played for ten minutes, the threshold score was increased again by 50 points (to 200 points), and so on until the ten minutes were reached. In average, each participant made $M=83.4$ trials $(S D=17.2)$, which had a duration of $M=7.2$ seconds $(S D=4.9)$.

Dependent measures. The first measure was the average score obtained by each participant across trials. All other measures were computed from eye movement data. The game window was divided into eight AOI (Figure 2). AOI 1 to 6 were horizontal, whereas the two other ones ("ScoreTop" and "ScoreBottom" AOI, size 123x89 pixels) covered the locations of the score display used in the "top" and "bottom" conditions, respectively. The ScoreTop AOI was cut out of AOI 1 (size 480x310 pixels), which was the area located above the ball's axis of movement. The space below was divided in five horizontal areas of interest of almost equal size numbered from top to bottom. The median line of AOI 2 (480x72 pixels) was the ball's horizontal axis of movement. The sizes of $A O I 3$ to 6 , where participants could anticipate the movement of obstacles, were the same as that of AOI 2, except for AOI 6 (480x74 pixels). The ScoreBottom AOI was cut out of $\mathrm{AOI} 5$ and 6 . The first gaze-related dependent variables were the total number of eye fixations made during the game and the number of fixations in each $\mathrm{AOI}$.

Two summary measures were computed from the vertical coordinates (along the $\mathrm{Y}$-axis) of the position of each ocular fixation to assess participants' anticipation of the movement of obstacles and extent of visual scanning. Participants' anticipation of the movement of obstacles was estimated through the mean vertical gaze position counted in pixels from the top (pixel 1) to the bottom (pixel 672) of the game window. Lower average gaze positions should indeed allow participants to detect the appearance of obstacles and anticipate their movement from the bottom of the screen more in advance. The extent of visual scanning during the game was evaluated through the standard deviation of the mean vertical eye position, which gave an idea of the vertical scattering of fixations. Higher standard deviations of the mean vertical eye position indicated that participants were scanning a larger part of the game screen. 


\section{RESULTS}

To optimize data analysis, trials were filtered using the two following criteria. The first twenty trials of each participant, approximately the first 2 minutes of effective play, were excluded from analysis as a training phase during which participants got to know the game and how to manipulate the keypad. Among the remaining trials (about 60 per participant), only those where a score of $\mathbf{2 0}$ or more was reached were retained for analysis. Indeed, letting the video game run by itself for 250 trials, without any human control of the ball, gave an average score of 14.6, SD = 3.6. Hence, scores lower than 20 were at chance level and gave no information on players' abilities. The average number of trials that were excluded because of low scores was $M=21.3(S D=10.5)$ for the "bottom" and $M=21.5(S D=13.9)$ for the "top" condition. Altogether, data analysis was performed on $M=43.1$ trials $(S D=7.0)$ per participant for the "bottom" and $M=40.9$ trials $(S D=7.4)$ for the "top" condition. Their duration was $M=9.3$ seconds $(S D=1.8)$ for the "bottom" and $M=9.5$ seconds $(S D=2.1)$ for the "top" condition.

Number of eye fixations. There was no significant difference between the number of fixations made by participants within the game window (pooling all AOI together) in the "top" $(M=1052, S D=302)$ and "bottom" $(M=1246, S D=$ 292) conditions, $t(28)=1.79, p=.08$. The distribution of eye fixations between the eight $A O I$ in each condition is given by Table 2. Whatever the condition, about $40 \%$ of eye fixations fell within AOI 3 and $20 \%$ each in AOI 2 (around the ball's axis of movement) and 4 . More than $70 \%$ of fixations were made in AOI 3 to 6 . Hence, participants fixated mostly on the AOI below the ball's horizontal axis of movement where they could anticipate the movement of upward moving squares.

Table 2. Mean number and proportion of eye fixations made by participants in each AOI in each condition of Experiment 1 . The numbers in brackets correspond to standard deviations.

"top" condition

\section{"bottom" condition}

\begin{tabular}{ccccc}
\hline AOI & Number of fixations & Proportion & Number of fixations & Proportion \\
$\mathbf{1}$ & $43(61)$ & $4.1 \%$ & $16(38)$ & $1.3 \%$ \\
$\mathbf{2}$ & $206(131)$ & $19.6 \%$ & $251(225)$ & $20.1 \%$ \\
$\mathbf{3}$ & $418(118)$ & $39.7 \%$ & $504(152)$ & $40.4 \%$ \\
$\mathbf{4}$ & $245(92)$ & $23.2 \%$ & $284(109)$ & $22.8 \%$ \\
$\mathbf{5}$ & $76(62)$ & $7.3 \%$ & $117(74)$ & $9.4 \%$ \\
$\mathbf{6}$ & $16(38)$ & $1.5 \%$ & $21(40)$ & $1.6 \%$ \\
ScoreTop & $43(20)$ & $4.1 \%$ & $0(0)$ & $0.0 \%$ \\
ScoreBottom & $5(6)$ & $0.1 \%$ & $54(30)$ & $4.3 \%$ \\
\hline
\end{tabular}

Independent-samples $t$ tests were used to compare the proportions of fixations made in each AOI between the two conditions. Participants made a higher proportion of fixations in AOI ScoreTop in the "top" than in the "bottom" condition, $t(28)=-8.53, p<.001$, Cohen's $d=3.12$. In contrast, participants made a higher proportion of fixations in AOI ScoreBottom in the "bottom" condition, $t(28)=6.30, p<.001, d=4.59$. There was no significant difference in the proportions of fixations that fell within the other AOI between the two conditions.

Vertical position and scattering of fixations. As shown on Figure 2, the mean vertical position of eye fixations was lower in the "bottom" $(M=501.9, S D=31.7)$ than in the "top" $(M=467.1, S D=29.2)$ condition, $t(28)=3.13, p<.01, d=1.14$. 
In addition, the mean vertical scattering of fixations was smaller in the "bottom" ( $M=70.2, S D=12.1)$ than in the "top" $(M=103.7, S D=16.5)$ condition, $t(28)=6.35, p<.001, d=2.32$.

Score performance. The score performance did not differ significantly between the "top" $(M=40.7, S D=7.7)$ and "bottom" ( $M=39.3, S D=8.9)$ conditions, $t(28)=.39, p=.70$. The mean maximal score was $96.0(S D=26.0)$. Five $(33 \%)$ and eight (53\%) participants out of 15 hit the initial threshold of 100 points in the "bottom" and "top" conditions, respectively. Only one participant playing in the "bottom" condition hit the subsequent 150 points threshold.

\section{DISCUSSION}

Preliminary hypothesis: visual scanning of the game display. The preliminary hypothesis was confirmed (see Table 2). Eye movement recordings showed that regardless of the score display location, more than $95 \%$ of participants' eye fixations were made either in the ball area ( $\mathrm{AOI} 2$, about $20 \%$ of fixations), in the zone of anticipation of the movement of obstacles ( $\mathrm{AOI} 3$ to 6,70 to $75 \%$ of fixations) or on the score display $\mathrm{AOI}$ (4 to $5 \%$ of fixations). The number of fixations within the ScoreTop and ScoreBottom AOI varied according to the score display location: Participants fixated the ScoreTop and ScoreBottom AOI when the score was displayed inside, i.e. in the "top" and "bottom" conditions, respectively. Hence, the score display was relevant for the game task and was monitored by participants while playing. According to the SEEV model, the high number of fixations within the anticipation zones of the screen may result from high user's expectancy towards these AOI. The score display would also be the focus of players' expectancy as well as value, and may have a higher salience than other elements of the display.

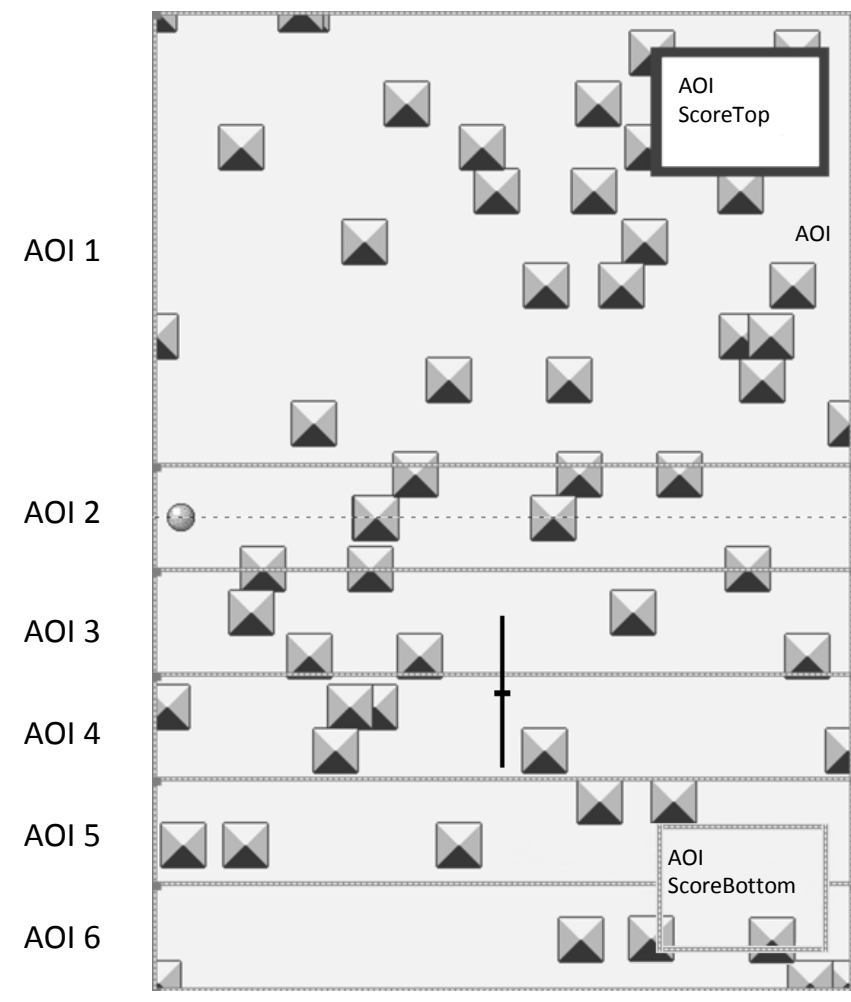

"top" condition

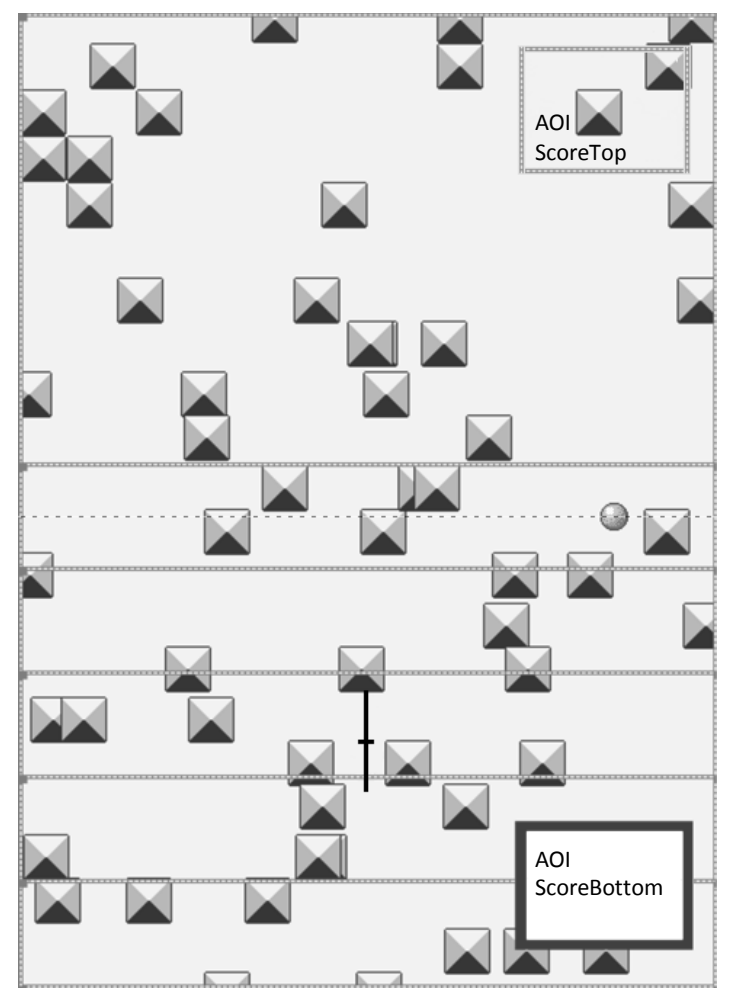

"bottom" condition

Figure 2. Screenshots of the video game used in Experiment 1 in the "top" and "bottom" conditions. The dark gray horizontal lines mark the limits of the horizontal areas of interest (AOI). The top one is AOI 1 and the bottom one AOI 6 . The white-filled black rectangles mark the limits of the score $\mathrm{AOI}$ where the score was located in each condition. Conversely, the grey rectangles indicate the limit of the empty score AOI. The black horizontal and vertical bars represent the mean vertical position and scattering of eye fixations in each condition. 
Reduced extent of visual scanning in the "bottom" condition. The second hypothesis was confirmed. Regardless of the location of the score display, more than $70 \%$ of fixations were made in the portion of the game window where the movement of obstacles could be anticipated. The third hypothesis was also verified. Participants' visual behavior was modified when the score was located at the bottom rather than the top of the game window. The mean vertical position of fixations was lower and their vertical scattering smaller in the "bottom" than in the "top" condition. Hence, positioning the score at the bottom of the game window focused and oriented participants' gaze towards the obstacles' point of origin by reducing the extent of visual scanning and instigating participants' inspection of the bottom of the screen.

Impact of reduced extent of visual scanning on performance. According to the PCP, higher spatial proximity between the score display and the anticipation zone should facilitate anticipation of obstacle movement and increase player performance. However, this fourth hypothesis was not supported. Minimizing the extent of visual scanning by displaying the score at the bottom of the game window did not result in a significant increase of players' performance compared to the "top" condition. This was despite the fact that a lower mean vertical position of fixations should have helped them to anticipate the movement of obstacles.

Three factors might explain why moving the score display closer to the anticipation zone did not increase performance. First, as pointed out in the description of the PCP, too high spatial proximity between visual signals that must be attended together can provoke intra-modal interference in visual perception, which can generate confusing or masking effects that may impair information integration (Wickens et al., 2004). Since in the "bottom" condition, the score was displayed within the game anticipation zone, the overlap may have impaired anticipation of the movement of obstacles.

Second, participants may have been able to share their visual attention resources between the focal and ambient channels to optimize anticipation of the movement of obstacles. The data showed that although the vertical position of fixations was lower in the "bottom" than in the "top" condition, this did not lead to better performance. This suggests that participants might use parafoveal vision efficiently to scan the anticipation zone even in the "top" condition.

Third, the threshold score of 100 points was difficult to reach for many participants. Seventeen participants played for ten minutes without achieving this goal. Video games are entertainment products that must be pleasant to play, and the challenge must be adjusted to players' abilities to be accepted. Here, the participants that had difficulties hitting the threshold scores may have felt that the game was too difficult and were subsequently "turned off". A "floor effect" might therefore explain why the score display manipulation did not significantly impact performance.

Rationale of Experiment 2. Informed by the results of Experiment 1, a new experiment was designed to further investigate the ways that reducing the extent of visual scanning may lead to better performance in video games, in alignment with the PCP.

Experiment 1 was modified in two ways. First, an attempt was made to reduce visual interference that may have resulted from the overlap of the score display with the game anticipation zone in the "bottom" condition. Two new score display locations were tested (Figure 1). The score could be located on the right of the game window just above the ball's axis of movement ("just above the ball" condition), or at the bottom right of the computer screen below the game window ("far bottom" condition) to induce participants to look where the obstacles came from as in the "bottom" condition. The rationale was to move the score display closer to the game anticipation zone than in the "top" condition, but not within this zone. Second, the initial threshold score for success was divided by 2.5 (from 100 to 40 ) to make the game easier, increase the motivation of the most novice players and get rid of eventual floor effects.

Hypotheses were also modified. Closer proximity between simultaneously attended visual signals would lead to better performance only when there is no visual interference or overlap between these signals. In dynamic environments, the optimal solution would be to minimize the extent of visual scanning between the relevant game areas only up to the extent there is no overlap between them. 
The first hypothesis of Experiment 2 was that anticipation, assessed by the mean vertical position of fixations, depends on the location of the score display relative to the anticipation zone. We expected that anticipation is greater in the "far bottom" and "bottom" conditions than in the "just above the ball" and "top" conditions. The second hypothesis was that the extent of visual scanning of the game display, assessed by the vertical scattering of fixations, is minimal in the "bottom" condition, intermediate (and optimal) in the "just above the ball" and "far bottom" conditions and maximal in the "top" condition. Hypothesis 3a was that game performance is the best for the "far bottom" condition, which associates the greatest anticipation with optimal extent of visual scanning. According to hypothesis $3 \mathrm{~b}$, the second best performance was expected for the "just above the ball" condition, which combines the same optimal extent of visual scanning as the "far bottom" condition with lower anticipation possibilities. In both cases, performance was expected to be greater than in the "top" condition, which fails to support anticipation, and the "bottom" condition, which potentially results in interference associated with overlap between the score display and the anticipation zone. Table 1 displays the main characteristics of the score display designs used in the four conditions of Experiment 2.

\section{EXPERIMENT 2}

\section{MethodS}

Participants. Twenty-one right-handed volunteers (12 women, 9 men) took part in the experiment. Their average age was 26.2 years $(S D=7.1)$ and their average length of schooling 16.1 years $(S D=2.8)$. Nine of them had never played video games, nine participants were occasional players and three were regular players.

Material and apparatus. The video game was the same as in Experiment 1, but two new locations were designated for the score display. The score was displayed on the right just above the axis of movement of the ball (within AOI 1 ) in the "just above the ball" condition, and on the right below the game window in the "far bottom" condition (Figure 1). The apparatus was the same as in the first experiment. The average distance between the eye-tracker screen and participants' eyes was $651 \mathrm{~mm}(S D=71)$, which means that 0.5 degree of visual angle corresponded to about $5.67 \mathrm{~mm}$ on the screen.

Design and procedure. The location of the score display was manipulated within-participants. The total time of effective play was increased to 20 minutes, with the score display randomly assigned to one of the four possible positions ("top", "bottom", "just above the ball" or "far bottom") at the beginning of each trial. Participants' goal was the same as in Experiment 1, but the initial threshold score was decreased to 40 points and the threshold score increased by 40 points (instead of 50) each time participants succeeded. The target threshold adjustment was valid for all subsequent trials independently of the score display condition in which the threshold score was reached. Participants were invited to take a short break approximately every five minutes of effective play. Participants completed an average of 176.7 trials $(S D=44.2)$, which had a duration of $M=7.4$ seconds $(S D=5.3)$.

Dependent measures. Experiment 2 used the same dependent measures as in Experiment 1, except that the number of fixations falling within each AOI was not used. Indeed, the "per AOI" analysis of Experiment 1 only revealed that the participants' glances to score-related information went to different parts of the screen in the "top" and "bottom" conditions. The more informative summary eye movement measures, namely the average vertical position and vertical scattering of fixations, appeared sufficient to estimate participants' visual anticipation and extent of visual scanning.

\section{REsults}

As in Experiment 1, the first twenty trials of each participant and all trials with scores lower than 20 were removed from analysis. For each participant, the number of trials that were excluded because of low scores went from $M=12.2$ (SD = 7.1) for the "top" to $M=14.8(S D=8.2)$ for the "far bottom" condition. Data analysis was thus performed on $M=26.0$ 
trials $(S D=6.5)$ per participant for the "top", $M=27.2$ trials $(S D=6.1)$ for the "bottom", $M=25.2$ trials $(S D=6.0)$ for the "just above the ball" and $M=25.8$ trials $(S D=6.4)$ for the "far bottom" condition. Their duration went from $M=9.1$ seconds $(S D=2.1)$ in the "top" condition to $M=10.2$ seconds $(S D=2.9)$ in the "far bottom" condition.

Data were analyzed using ANOVAs with the score location as a within-participants factor. When the Mauchley sphericity test was significant, Greenhouse-Geisser correction was applied.

Vertical position and scattering of fixations. There was an effect of the score display location on the vertical position of fixations, $F(2,38)=17.65, p<.001, \eta_{p}^{2}=.469$ after Greenhouse-Geisser correction (Figures 3 and 4). Planned comparisons showed that the vertical position of fixations was marginally lower when the score was displayed at the bottom $(M=467.6, S D=45.5)$ rather than below the game window $(M=464.7, S D=43.5), F(1,20)=4.02, p=.059$. The average vertical position of fixations was lower in both the "bottom" and "far bottom" than in the "just above the ball" $(M=453.8, S D=44.1)$ or "top" $(M=453.3, S D=46.2)$ conditions, $F s(1,20)>12.16, p<.01$, but did not differ significantly between the two latter conditions, $F(1,20)=.04, p=.84$.

Figures 3 and 4 show that the vertical scattering of fixations was also impacted by the position of the score display, $F(2$, $32)=23.80 p<.001, \eta_{p}^{2}=.543$ after Greenhouse-Geisser correction. Vertical scattering of fixations was larger in the "top" $(M=87.8, S D=14.4)$ than in the three other conditions, $F s(1,20)>16.62, p<.001$. Vertical scattering of fixations was larger in the "far bottom" $(M=77.9, S D=9.9)$ than in the "bottom" $(M=73.1, S D=8.3)$ and "just above the ball" $(M=74.9, S D=10.1)$ conditions, $F s(1,20)>7.12, p<.05$, but was not significantly different between the two latter conditions, $F(1,20)=2.34, p=.14$.
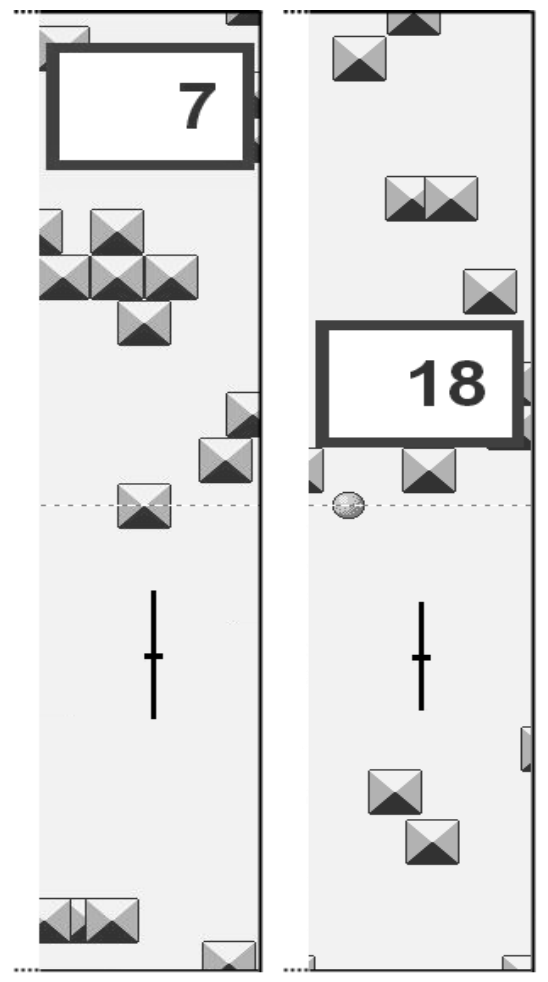

Top Just above the ball
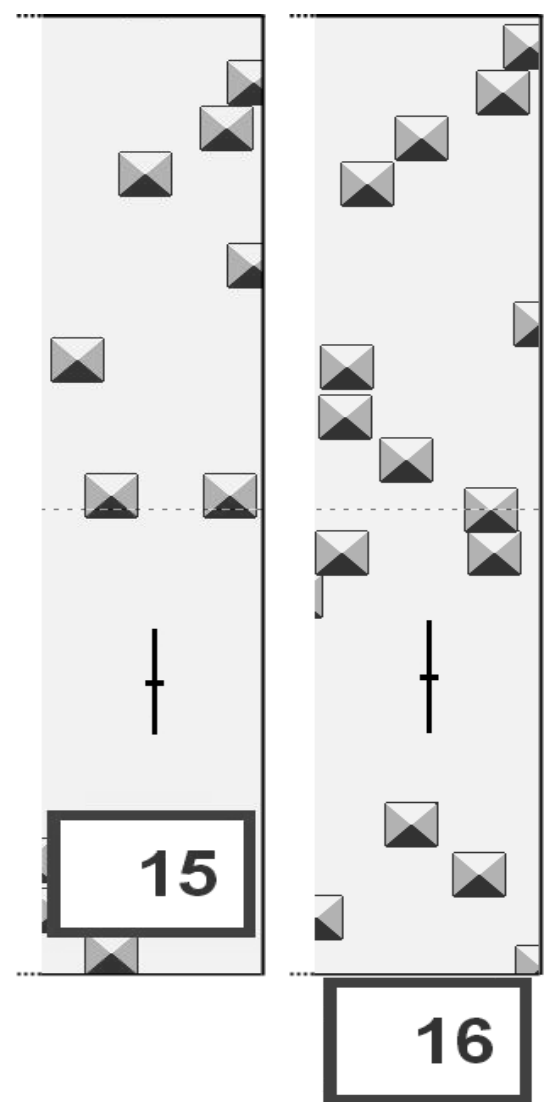

Far bottom

Figure 3. Right sides of screenshots of the video game used in Experiment 2 in the four score display conditions. The black horizontal and vertical bars represent the mean vertical position and scattering of eye fixations in each condition. 


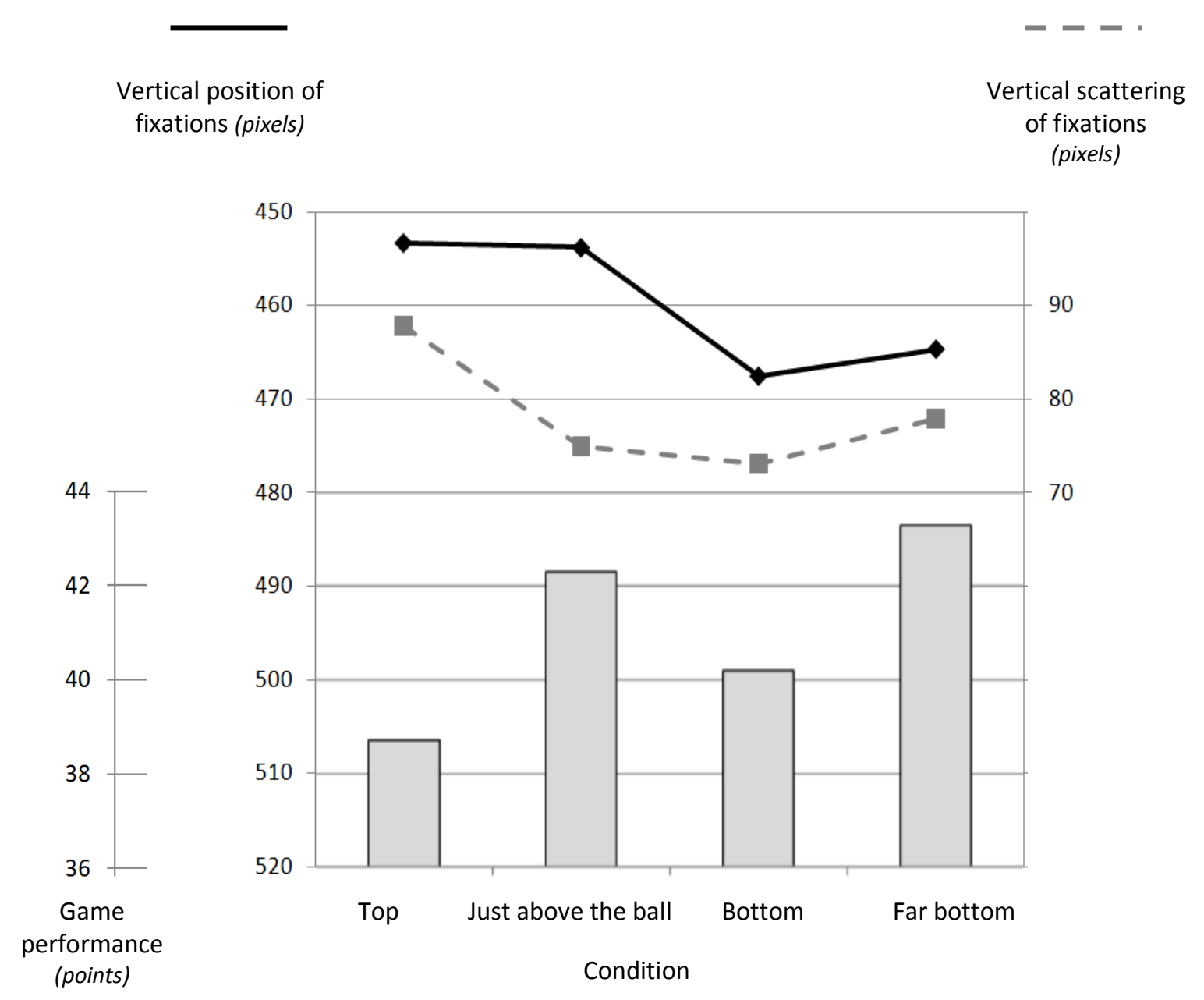

Figure 4. Graph showing the average measures obtained for the three dependent variables in each condition of Experiment 2. The bars represent participants' mean game performance.

Score performance. Figure 4 indicates that there was an effect of the position of the score display on performance, $F(3$, $60)=2.90, p<.05, \eta_{p}^{2}=.127$. Better or marginally better scores were obtained in the "far bottom" $(M=43.3, S D=12.5)$ than in the "top" $(M=38.7, S D=8.7), F(1,20)=6.83, p<.05$ or "bottom" $(M=40.2, S D=9.4), F(1,20)=4.04, p=.058$ conditions. The score performance was not significantly different between the "far bottom" and "just above the ball" $(M=42.3, S D=14.6)$ conditions, $F(1,20)=.26, p=.62$. No other comparison reached statistical significance. The mean maximal score was $123.9(S D=45.4)$. All 21 participants reached the initial 40 points threshold in 20 minutes, nine of them (43\%) hit the third 120 points threshold and two of them (10\%) the fifth 200 points threshold.

\section{DISCUSSION}

As expected, the average vertical position of fixations was lower in the "far bottom" and "bottom" than in the "just above the ball" and "top" conditions. The mean vertical position of fixations was not significantly different between the two latter conditions, but was marginally lower in the "bottom" than in the "far bottom" condition. This may be due to participants using their lower parafoveal visual field to process more of the lowest anticipation area (AOI 6) and 
the score display in the "far bottom" than in the "bottom" condition. Indeed, a larger effort was needed to fixate the score in the former situation.

The second hypothesis, namely that the vertical scattering of fixations should be minimal in the "bottom" condition, intermediate in the "just above the ball" and "far bottom" conditions and maximal in the "top" condition, was not verified. As expected, the vertical scattering of eye fixations was the largest for the "top" condition and was greater in the "far bottom" than in the "bottom" condition. However, vertical scattering in the "just above the ball" condition was lower than in the "far bottom" condition and not significantly different from the "bottom" condition. One explanation may be that the score display was closer from the average vertical position of fixations in the "just above the ball" and "bottom" than in the "far bottom" condition (Figures 3 and 4). Indeed, the mean vertical position of fixations did not vary as much as in Experiment 1 across conditions, and always stayed at or just below the border of AOI 3 and 4 .

Most importantly, hypothesis 3a was verified. Participants' scores were higher in the "far bottom" condition than in the two conditions of Experiment 1. The absence of a significant difference between the scores obtained in the "top" and "bottom" conditions replicated the Experiment 1 findings. However, hypothesis $3 \mathrm{~b}$ was not verified because there was no significant difference between the "far bottom" and "just above the ball" condition. In the latter case, participants' scores were intermediate between those of the "far bottom" and of the "top and "bottom" conditions. The expected advantage of the "far bottom" over the "just above the ball" condition may has been reduced because even if the average vertical position of fixations was lower in the "far bottom" condition, the extent of visual scanning was lower in the "just above the ball" condition.

The main theoretical hypothesis that guided the conception of Experiment 2 was essentially supported. In the dynamic context of video games, closer proximity between simultaneously attended visual information would favor integration only if there is no overlap between the relevant visual areas of the game screen. The optimal extent of visual scanning would be obtained by presenting the different pieces of information side by side rather than presenting them with even partial overlap.

\section{GENERAL Discussion}

The PCP recommends that display designers place in close proximity information sources that share common endpoints, i.e. those that contribute to the realization of the same task or mental operation. This design rule would minimize the effort needed to move attention between the two source locations.

The two present experiments were based on a video game where the main area of attention was a visual anticipation zone located in the bottom part of the game window. In Experiment 1, the score display was located either at the top, as is usual in this kind of game, or at the bottom of the window. Since the top location was distal from the visual anticipation zone, the PCP would rather recommend putting the score display at the bottom of the game window to minimize attentional moves between this area and the score display. The results of Experiment 1 did show lower fixations and decreased extent of visual scanning in the bottom condition, but contrary to what was expected, the performance did not improve significantly.

In Experiment 2, two more score display locations were tested, namely the "just above the ball" and "far bottom" locations. Both were chosen to be close to the visual anticipation zone as recommended by the PCP, but not within this zone according to the results of Experiment 1. Experiment 2 demonstrated that when the score was displayed below the game window in the "far bottom" condition, both players' visual anticipation and performance increased significantly compared to the "top" condition despite a significant increase of the extent of visual scanning compared to the "bottom" condition. Altogether, even though attending to the score was part of the game task, better performance was obtained when there was no overlap between the score location and the visual anticipation zone.

Two explanations can be provided. The first one supposes that the PCP would apply to dynamic video games, but only in contexts where there is an absence of visual overlap between the anticipation zone and the information that must be 
simultaneously assessed. Close spatial proximity between these pieces of information would facilitate divided attention only as long as there is no interference-inducing overlap. In the case of overlap, the inability to focus attention on only one of the two information sources would become detrimental for performance. Experimental data show that players could better anticipate the moves of obstacles when the score display was at the bottom of or just below the game window. The fact that performance was better in the "far bottom" than in the "bottom" condition, even though the extent of visual scanning was larger, suggests that minimizing the visual overlap between information sources is more beneficial for players' performance than reducing visual scanning.

In the multiple resources model (Wickens, 2002; Wickens \& McCarley, 2008), the visual modality includes two different attentional channels, namely the focal and ambient ones. In the "far bottom" condition, the focal channel might be used to anticipate the movement of obstacles while the ambient channel would follow the score display information. This interpretation is somewhat counter-intuitive because the focal channel is considered more suited for object recognition than the ambient channel. However, precise anticipation of the movement of obstacles might be a more demanding attentional task than briefly attending a large-sized score display. The large size and high familiarity of score digits should indeed make score identification via the peripheral visual field easier. Several authors actually demonstrated that words could be read and recognized within the peripheral visual field through "covert attention" processes (Lee, Legge, \& Ortiz, 2003; Pelli, Tillman, Freeman, Su, Berger, \& Majaj, 2007).

An alternative explanation emerges when considering the PCP continuum (Wickens \& Carswell, 1995; Wickens \& McCarley, 2008). This principle emphasizes that the more information sources are used simultaneously for the same task, the more they should be placed close to each other. Here, the two information sources (the movement of obstacles and the score display) may be insufficiently integrated during the task to warrant the strong proximity that characterized the "bottom" condition. Players had to follow the score display while playing to see when they reached the assigned threshold. However, knowing the score did not help them to avoid the obstacles, and the task proximity between the two information sources can be considered as rather low. Hence, putting the two information sources very close to each other may not be necessary for optimal performance. Placing them moderately close to each other (like in the "far bottom" and "just above the ball" conditions) rather than at different ends of the screen ("top" condition) may be sufficient. Whatever the explanation, the PCP and multiple resources model provide a sufficient frame to make predictions and interpret the data.

In conclusion, for the video game used in this study, positioning the score display at the "far bottom" location helped the players to anticipate the movement of obstacles and to obtain a better performance. This result may be generalizable to all others video games that require that players visually anticipate movements. For video game designers, a recommendation could be that for such games, the main elements of contextual information should be located in the expected direction of anticipation, but should not overlap with the main action and anticipation zones.

The results of the current study complement the PCP of Wickens and Carswell (1995) for the design of simple dynamic visual displays. In addition, the above heuristic might be generalized to all interactive environments that require anticipation from human operators, for instance to the design of car driving or plane piloting posts. Further studies are however required to check the validity of this heuristic in more complex systems.

\section{ACKNOWLEDGMENTS}

Loïc Caroux was supported by a Ph.D. fellowship from the Direction Générale de l'Armement (DGA, French Ministry of Defense), and followed in that frame by Didier Bazalgette. 


\section{REFERENCES}

Ang, C. S., Zaphiris, P., \& Mahmood, S. (2007). A model of cognitive loads in massively multiplayer online role playing games. Interacting with Computers, 19, 167-179.

Barr, P., Noble, J., \& Biddle, R. (2007). Video game values: Human-computer interaction and games. Interacting with Computers, 19, 180-195.

Boot, W. R., Kramer, A. F., Simons, D. J., Fabiani, M., \& Gratton, G. (2008). The effects of video game playing on attention, memory, and executive control. Acta Psychologica, 129, 387-398.

Green, C. S., \& Bavelier, D. (2003). Action video game modifies visual selective attention. Nature, 423, 534-537.

Horrey, W. J., Wickens, C. D., \& Consalus, K. P. (2006). Modeling drivers' visual attention allocation while interacting with in-vehicle technologies. Journal of Experimental Psychology-Applied, 12, 67-78.

Lee, H.-W., Legge, G. E., \& Ortiz, A. (2003). Is word recognition different in central and peripheral vision ? Vision Research, 43, $2837-$ 2846.

Nielsen, J. (1993). Usability engineering. Boston: Academic press.

Pagulayan, R. J., Keeker, K., Wixon, D., Romero, R. L., \& Fuller, T. (2008). User-centered design in games. In A. Sears \& J. A. Jacko (Eds.), The Human-Computer Interaction Handbook: Fundamentals, Evolving Technologies, and Emerging Applications (pp. 741 759). New York: Lawrence Erlbaum Associates.

Pelli, D. G., Tillman, K. A., Freemen, J., Su, M., Berger, T. D., \& Majaj, N. J. (2007). Crowding and eccentricity determine reading rate. Journal of Vision 7(2), 20, 1-36.

Pierno, A. C., Caria, A., Glover, S., \& Castiello, U. (2005). Effects of increasing visual load on aurally and visually guided target acquisition in a virtual environment. Applied Ergonomics, 36, 335-343.

Rayner, K. (1998). Eye movements in reading and information processing: 20 years of research. Psychological Bulletin, 124, $372-422$.

Renshaw, J. A., Finlay, J. E., Tyfa, D., \& Ward, R. D. (2004). Understanding visual influence in graph design through temporal and spatial eye movement characteristics. Interacting with Computers, 16, 557-578.

Stanney, K. M., Mollaghasemi, M., Reeves, L., Breaux, R., \& Graeber, D. A. (2003). Usability engineering of virtual environments (VEs): identifying multiple criteria that drive effective VE system design. International Journal of Human-Computer Studies, 58, 447-481.

Underwood, G. (2007). Visual attention and the transition from novice to advanced driver. Ergonomics, 50, 1235-1249.

Underwood, G., Chapman, P., Brocklehurst, N., Underwood, J., \& Crundall, D. (2003). Visual attention while driving: sequences of eye fixations made by experienced and novice drivers. Ergonomics, 46, 629-646.

Wickens, C. D. (1984). Processing resources in attention. In R. Parasuraman \& D. R. Davies (Eds.), Varieties of attention (pp. 62-102). New York: Academic Press.

Wickens, C. D. (2002). Multiple resources and performance prediction. Theoretical Issues in Ergonomics Science, 3, 159-177.

Wickens, C. D., Alexander, A. L., Ambinder, M. S., \& Martens, M. (2004). The role of highlighting in visual search through maps Spatial Vision, 17, 373-388.

Wickens, C. D., \& Carswell, C. M. (1995). The proximity compatibility principle: Its psychological foundation and relevance to display design. Human Factors, 37, 473-494.

Wickens, C. D., Goh, J., Helleberg, J., Horrey, W. J., \& Talleur, D. A. (2003). Attentional models of multitask pilot performance using advanced display technology. Human Factors, 45, 360-380.

Wickens, C. D., \& McCarley, J. S. (2008). Applied attention theory. Boca-Raton, FL: Taylor \& Francis. 